\title{
A discussion on a generalized Geraghty multi-valued mappings and applications
}

\author{
Hojjat Afshari , Maryam Atapour ${ }^{1}$ and Erdal Karapınar ${ }^{2,3,4,5^{*}}$ (D)
}

\author{
"Correspondence: \\ erdalkarapinar@duytan.edu.vn; \\ erdalkarapinar@yahoo.com \\ ${ }^{2}$ Institute of Research and \\ Development, Duy Tan University, \\ 550000 Da Nang, Vietnam \\ ${ }^{3}$ Faculty of Natural Sciences, Duy \\ Tan University, 550000 Da Nang, \\ Vietnam \\ Full list of author information is \\ available at the end of the article
}

\begin{abstract}
This research intends to investigate the existence results for both coincidence points and common fixed point of generalized Geraghty multi-valued mappings endowed with a directed graph. The proven results are supported by an example. We also consider fractional integral equations as an application.
\end{abstract}

Keywords: Directed graph; Generalized Geraghty multi-valued mappings; Coincidence point; Fractional integral equation

\section{Introduction and preliminaries}

The metric fixed point theory, which has wide common application potential in distinct quantitative sciences, develops itself in parallel with the developments in applied areas $[8,14-16]$. One of the examples of this trend was given by Echenique [12] who considered metric fixed point theory in the framework of graphs. Later, Espinola and Kirk [13] applied some well-known fixed point results and techniques to the graph theory. On the other hand, in 2008, Jachymski [17] proposed a new contraction, the concept graph contraction, in order to generalize the distinguished Banach contraction principle.

In this paper, we shall consider generalized Geraghty multi-valued mappings in the context of complete metric spaces endowed with a graph. After investigating the existence and uniqueness of a fixed point for such mappings, we shall consider a fractional integral equation $[1-7,9,10,18-21,23-25,27]$ and we solve this equation via our obtained results.

Throughout the paper, we presume that all considered sets and subsets are non-empty. The pair $(M, d)$ denotes a metric space and $\left(M^{*}, d\right)$ represent complete metric space. Let $\Delta$ be the diagonal of $M \times M$. Assume that $\Gamma$ is a directed graph with vertex set $V(\Gamma)$ which is coincided with $M$ and edge set $E(\Gamma)$ which contains $\Delta$. Suppose there is not any parallel edges in $G$. We shall say that $g: M \rightarrow M$ is a Banach $G$-contraction if:

(i) $(x, y) \in E(\Gamma)$ implies $(g(x), g(y)) \in E(\Gamma)$, for all $x, y \in M$,

(ii) $\exists 0<\alpha<1$ such that, for $x, y \in M$,

$$
(x, y) \in E(\Gamma) \quad \Rightarrow \quad d(g(x), g(y)) \leq \alpha d(x, y) .
$$

(c) The Author(s) 2020. This article is licensed under a Creative Commons Attribution 4.0 International License, which permits use, sharing, adaptation, distribution and reproduction in any medium or format, as long as you give appropriate credit to the original author(s) and the source, provide a link to the Creative Commons licence, and indicate if changes were made. The images or other third party material in this article are included in the article's Creative Commons licence, unless indicated otherwise in a credit line to the material. If material is not included in the article's Creative Commons licence and your intended use is not permitted by statutory regulation or exceeds the permitted use, you will need to obtain permission directly from the copyright holder. To view a copy of this licence, visit http://creativecommons.org/licenses/by/4.0/. 
Assume that $(M, d)$ be a metric space and $P_{b, c l}(M)$ be the family of bounded and closed sets in $M$. For $x \in M$ and $M_{1}, M_{2} \in P_{b, c l}(M)$,

$$
\begin{aligned}
& D\left(x, M_{1}\right)=\inf _{a \in M_{1}} d(x, a), \\
& D\left(M_{1}, M_{2}\right)=\sup _{a \in M_{1}} D\left(M_{1}, M_{2}\right) .
\end{aligned}
$$

Define $H: P_{b, c l}(M) \times P_{b, c l}(M) \rightarrow[0, \infty)$ with

$$
H\left(M_{1}, M_{2}\right)=\max \left\{\sup _{x \in M_{1}} d\left(x, M_{2}\right), \sup _{y \in M_{2}} d\left(y, M_{2}\right)\right\},
$$

for $M_{1}, M_{2} \in P_{b, c l}(M) . H$ is the famous Hausdorff metric.

Lemma 1.1 ([11]) Let $(M, d)$ be a metric space. For $M_{1}, M_{2} \in P_{b, c l}(M)$ and $x, y \in M$, the following relations hold:

(1) $D\left(x, M_{2}\right) \leq d(x, b), b \in M_{2}$,

(2) $D\left(x, M_{2}\right) \leq H\left(M_{1}, M_{2}\right)$,

(3) $D\left(x, M_{1}\right) \leq d(x, y)+D\left(y, M_{1}\right)$,

(4) for all $\omega \in M_{1}$, there exists some $\varphi \in M_{2}$ such that $d(\omega, \varphi) \leq q H\left(M_{1}, M_{2}\right)$, where $q>1$.

Definition $1.2([28])$ For a set $M$, let $\Gamma=(V(\Gamma), E(\Gamma))$ be a graph with $V(\Gamma)=M$. Then:

(i) a mapping $T: M \rightarrow P_{b, c l}(M)$ is said to be graph preserving if it preserves the edges, i.e.,

$$
\text { if }(x, y) \in E(\Gamma) \text {, then }(u, v) \in E(\Gamma) \text { for all } u \in T x \text { and } v \in T y \text {, }
$$

(ii) mappings $S, T: M \rightarrow P_{b, c l}(M)$ are said to be mixed graph preserving respect to $h_{1}$, $h_{2}$ if the preserve the edges, i.e., for $x, y \in M$, if $\left(h_{1}(x), h_{2}(y)\right) \in E(\Gamma)$, then $(x, \tau) \in E(\Gamma)$ for all $x \in T x$ and $\tau \in S y$ and

$$
\text { if }\left(h_{2}(x), h_{1}(y)\right) \in E(\Gamma) \text {, then }(b, r) \in E(\Gamma) \text { for all } b \in S x \text { and } r \in T y \text {. }
$$

Consider the class

$$
\begin{aligned}
\Psi:= & \{\psi:[0, \infty) \rightarrow[0, \infty), \text { increasing, continuous, and } \psi(c t) \leq c \psi(t), \\
& \text { for all } c>1, \text { with } \psi(0)=0\}
\end{aligned}
$$

Let $\mathcal{F}$ denote the family of all functions $\beta:[0, \infty) \rightarrow[0,1)$.

\section{Main results}

We shall start this section by introducing the notion of the generalized Geraghty-type G-multi-valued mapping.

Definition 2.1 On a metric space $(M, d)$, let $\Gamma=(V(\Gamma), E(\Gamma))$ be a graph with vertex set $V(\Gamma)=M$ and the set $E(\Gamma)$ of its edges such that $E(\Gamma) \supseteq \Delta$. For $h_{1}, h_{2}: M \rightarrow M$ and 
$S, T: M \rightarrow P_{b, c l}(M) ; S, T$ are called a generalized $h_{1}, h_{2}$-Geraghty-type $G$-multi-valued mapping provided that

(i) $S, T$ are mixed graph preserving respect to $h_{1}, h_{2}$;

(ii) for $x, y \in M$ with $\left(h_{1}(x), h_{2}(y)\right) \in E(\Gamma)$, there exists $L \geq 0$ such that, for

$$
\begin{aligned}
P\left(h_{1}(x), h_{2}(y)\right)= & \max \left\{d\left(h_{1}(x), h_{2}(y)\right), D\left(h_{1}(x), T x\right), D\left(h_{2}(y), S y\right),\right. \\
& \left.\frac{D\left(h_{1}(x), T x\right)+D\left(h_{2}(y), S y\right)}{2}\right\} \text { and } \\
Q\left(h_{1}(x), h_{2}(y)\right)= & \min \left\{D\left(h_{1}(x), S y\right), D\left(h_{2}(y), T x\right)\right\}
\end{aligned}
$$

we have

$$
\begin{aligned}
\psi(H(S x, T y)) \leq & \gamma\left(\psi\left(P\left(h_{1}(x), h_{2}(y)\right)\right)\right) \psi\left(P\left(h_{1}(x), h_{2}(y)\right)\right) \\
& +L \phi\left(Q\left(h_{1}(x), h_{2}(y)\right)\right)
\end{aligned}
$$

and if $\left(h_{2}(x), h_{1}(y)\right) \in E(\Gamma)$ then

$$
\begin{aligned}
\psi(H(S x, T y)) \leq & \gamma\left(\psi\left(P\left(h_{2}(x), h_{1}(y)\right)\right)\right) \psi\left(P\left(h_{2}(x), h_{1}(y)\right)\right) \\
& +L \phi\left(Q\left(h_{2}(x), h_{1}(y)\right)\right)
\end{aligned}
$$

where $\gamma \in \mathcal{F}$ and $\psi, \phi \in \psi$.

Theorem 2.2 Let $(M, d)$ be a complete metric space endowed with a graph $\Gamma=(V(\Gamma)$, $E(\Gamma)), h_{1}, h_{2}: M \rightarrow M$ are surjective and $S, T: M \rightarrow P_{b, c l}(M)$ generalized $h_{1}, h_{2}$-Geraghtytype G-multi-valued mapping in $(M, d)$. Suppose

(i) $\exists x_{0} \in M$ such that $\left(h_{1}\left(x_{0}\right), u\right) \in E(\Gamma)$ for some $u \in T x_{0}$,

(ii) if $\left(h_{1}(x), h_{2}(y)\right) \in E(\Gamma)$, then $(e, f) \in E(\Gamma)$ for all $e \in T x$ and $f \in$ Sy and if $\left(h_{2}(x), h_{1}(y)\right) \in E(\Gamma)$, then $(w, r) \in E(\Gamma)$ for all $w \in S x$ and $r \in T y$,

(iii) for $\left\{x_{n}\right\}_{n \in \mathbb{N}}$ in $M$, if $x_{n} \rightarrow x$ and $\left(x_{n}, x_{n+1}\right) \in E(\Gamma)$ for $n \in \mathbb{N}$, then there is a subsequence $\left\{x_{n_{k}}\right\}_{n_{k} \in \mathbb{N}}$ such that $\left(x_{n_{k}}, x\right) \in E(\Gamma)$ for $n_{k} \in \mathbb{N}$.

Then there exist $u, v \in M$ such that $h_{1}(u) \in$ Tu or $h_{2}(v) \in S v$.

Proof Regarding that $h_{2}$ is surjective, one can find $x_{1} \in M$ such that $h_{2}\left(x_{1}\right) \in T x_{0}$ and $\left(h_{1}\left(x_{0}\right), h_{2}\left(x_{1}\right)\right) \in E(\Gamma)$. Let $q=\frac{1}{\sqrt{\gamma\left(\psi\left(d\left(h_{1}\left(x_{0}\right), h_{2}\left(x_{1}\right)\right)\right)\right)}}$. Then $q>1$, so

$$
0<D\left(h_{2}\left(x_{1}\right), S x_{1}\right) \leq H\left(T x_{0}, S x_{1}\right)<q H\left(T x_{0}, S x_{1}\right) .
$$

By Lemma 1.1 and (ii), $h_{1}$ is surjective, which implies that there exists $x_{2} \in M$ with $h_{1}\left(x_{2}\right) \in$ $S x_{1}$ and $\left(h_{2}\left(x_{1}\right), h_{1}\left(x_{2}\right)\right) \in E(\Gamma)$, hence

$$
\begin{aligned}
\psi\left(d\left(h_{2}\left(x_{1}\right), h_{1}\left(x_{2}\right)\right)\right)< & \psi\left(q H\left(T x_{0}, S x_{1}\right)\right) \leq q \psi\left(H\left(T x_{0}, S x_{1}\right)\right) \\
\leq & q \gamma\left(\psi\left(P\left(h_{1}\left(x_{0}\right), h_{2}\left(x_{1}\right)\right)\right)\right) \psi\left(P\left(h_{1}\left(x_{0}\right), h_{2}\left(x_{1}\right)\right)\right) \\
& +q L \phi\left(Q\left(h_{1}\left(x_{0}\right), h_{2}\left(x_{1}\right)\right)\right),
\end{aligned}
$$


where

$$
\begin{aligned}
P\left(h_{1}\left(x_{0}\right), h_{2}\left(x_{1}\right)\right)= & \max \left\{d\left(h_{1}\left(x_{0}\right), h_{2}\left(x_{1}\right)\right), D\left(h_{1}\left(x_{0}\right), T x_{0}\right), D\left(h_{2}\left(x_{1}\right), S x_{1}\right),\right. \\
& \left.\frac{D\left(h_{1}\left(x_{0}\right), T x_{0}\right)+D\left(h_{2}\left(x_{1}\right), S x_{1}\right)}{2}\right\} \\
= & \max \left\{d\left(h_{1}\left(x_{0}\right), h_{2}\left(x_{1}\right)\right), d\left(h_{2}\left(x_{1}\right), h_{1}\left(x_{2}\right)\right)\right\}
\end{aligned}
$$

and

$$
\begin{aligned}
Q\left(h_{1}\left(x_{0}\right), h_{2}\left(x_{1}\right)\right) & =\min \left\{D\left(h_{1}\left(x_{0}\right), S x_{0}\right), D\left(h_{2}\left(x_{1}\right), T x_{0}\right)\right\} \\
& \leq \min \left\{d\left(h_{1}\left(x_{0}\right), h_{2}\left(x_{1}\right)\right), d\left(h_{2}\left(x_{1}\right), h_{2}\left(x_{1}\right)\right)\right\}=0 .
\end{aligned}
$$

If we have

$$
\max \left\{d\left(h_{1}\left(x_{0}\right), h_{2}\left(x_{1}\right)\right), d\left(h_{2}\left(x_{1}\right), h_{1}\left(x_{2}\right)\right)\right\}=d\left(h_{2}\left(x_{1}\right), h_{1}\left(x_{2}\right)\right),
$$

then by (5) we get

$$
\begin{aligned}
\psi\left(d\left(h_{2}\left(x_{1}\right), h_{1}\left(x_{2}\right)\right)\right)< & \psi\left(q H\left(T x_{0}, S x_{1}\right)\right) \leq q \psi\left(H\left(T x_{0}, S x_{1}\right)\right) \\
\leq & q \gamma\left(\psi\left(P\left(h_{1}\left(x_{0}\right), h_{2}\left(x_{1}\right)\right)\right)\right) \psi\left(P\left(h_{1}\left(x_{0}\right), h_{2}\left(x_{1}\right)\right)\right) \\
& +q L \phi\left(Q\left(h_{1}\left(x_{0}\right), h_{2}\left(x_{1}\right)\right)\right) \\
= & q \gamma\left(\psi\left(d\left(h_{2}\left(x_{1}\right), h_{1}\left(x_{2}\right)\right)\right)\right) \psi\left(d\left(h_{2}\left(x_{1}\right), h_{1}\left(x_{2}\right)\right)\right) \\
= & \sqrt{\gamma\left(\psi\left(d\left(h_{2}\left(x_{1}\right), h_{1}\left(x_{2}\right)\right)\right)\right)} \psi\left(d\left(h_{2}\left(x_{1}\right), h_{1}\left(x_{2}\right)\right)\right) \\
< & \psi\left(d\left(h_{2}\left(x_{1}\right), h_{1}\left(x_{2}\right)\right)\right),
\end{aligned}
$$

a contradiction.

Hence, we obtain $\max \left\{d\left(h_{1}\left(x_{0}\right), h_{2}\left(x_{1}\right)\right), d\left(h_{2}\left(x_{1}\right), h_{1}\left(x_{2}\right)\right)\right\}=d\left(h_{1}\left(x_{0}\right), h_{2}\left(x_{1}\right)\right)$ and so by $(5)$

$$
\psi\left(d\left(h_{2}\left(x_{1}\right), h_{1}\left(x_{2}\right)\right)\right) \leq \sqrt{\gamma\left(\psi\left(d\left(h_{1}\left(x_{0}\right), h_{2}\left(x_{1}\right)\right)\right)\right)} \psi\left(d\left(h_{1}\left(x_{0}\right), h_{2}\left(x_{1}\right)\right)\right) .
$$

Keeping $\psi \in \psi$, in mind together with $\sqrt{\gamma\left(\psi\left(d\left(h_{1}\left(x_{0}\right), h_{2}\left(x_{1}\right)\right)\right)\right)}<1$, we get

$$
\begin{aligned}
& \psi\left(\frac{1}{\sqrt{\gamma\left(\psi\left(d\left(h_{1}\left(x_{0}\right), h_{2}\left(x_{1}\right)\right)\right)\right)}} d\left(h_{2}\left(x_{1}\right), h_{1}\left(x_{2}\right)\right)\right) \\
& \quad \leq \frac{1}{\sqrt{\gamma\left(\psi\left(d\left(h_{1}\left(x_{0}\right), h_{2}\left(x_{1}\right)\right)\right)\right)}} \psi\left(d\left(h_{2}\left(x_{1}\right), h_{1}\left(x_{2}\right)\right)\right) \\
& \quad<\psi\left(d\left(h_{1}\left(x_{0}\right), h_{2}\left(x_{1}\right)\right)\right) .
\end{aligned}
$$

Since $\psi$ is increasing, we have

$$
d\left(h_{2}\left(x_{1}\right), h_{1}\left(x_{2}\right)\right) \leq \sqrt{\gamma\left(\psi\left(d\left(h_{1}\left(x_{0}\right), h_{2}\left(x_{1}\right)\right)\right)\right)} d\left(h_{1}\left(x_{0}\right), h_{2}\left(x_{1}\right)\right) .
$$


Recall that $h_{1}\left(x_{2}\right) \in S x_{1}$ and $h_{2}\left(x_{1}\right) \in T x_{0}$. Choose

$$
q_{1}=\frac{\sqrt{\gamma\left(\psi\left(d\left(h_{1}\left(x_{0}\right), h_{2}\left(x_{1}\right)\right)\right)\right)} \psi\left(d\left(h_{1}\left(x_{0}\right), h_{2}\left(x_{1}\right)\right)\right)}{\psi\left(d\left(h_{2}\left(x_{1}\right), h_{1}\left(x_{2}\right)\right)\right)} .
$$

By (8), we have $q_{1}>1$. If $h_{1}\left(x_{2}\right) \in T x_{2}$, then $x_{2}$ forms a coincidence point for $h_{1}$ and $T$. We presume that $h_{1}\left(x_{2}\right) \notin T x_{2}$. We get

$$
0<\psi\left(d\left(h_{1}\left(x_{2}\right), T x_{2}\right)\right) \leq \psi\left(H\left(S x_{1}, T x_{2}\right)\right)<q_{1} \psi\left(H\left(S x_{1}, T x_{2}\right)\right) .
$$

Hence, there exists $h_{2}\left(x_{3}\right) \in T x_{2}$ such that $\left(h_{1}\left(x_{2}\right), h_{2}\left(x_{3}\right)\right) \in E(\Gamma)$ and

$$
\begin{aligned}
\psi\left(d\left(h_{1}\left(x_{2}\right), h_{2}\left(x_{3}\right)\right)\right)< & q_{1} \psi\left(H\left(S x_{1}, T x_{2}\right)\right) \\
\leq & q_{1} \gamma\left(\psi\left(P\left(h_{1}\left(x_{1}\right), h_{2}\left(x_{2}\right)\right)\right)\right) \psi\left(P\left(h_{1}\left(x_{1}\right), h_{2}\left(x_{2}\right)\right)\right) \\
& +q_{1} L \phi\left(Q\left(h_{1}\left(x_{1}\right), h_{2}\left(x_{2}\right)\right)\right) .
\end{aligned}
$$

Similarly, $P\left(h_{1}\left(x_{1}\right), h_{2}\left(x_{2}\right)\right) \leq d\left(h_{1}\left(x_{1}\right), h_{2}\left(x_{2}\right)\right)$ and $Q\left(h_{1}\left(x_{1}\right), h_{2}\left(x_{2}\right)\right)=0$. By $(8)$ and a property of $(\gamma)$, we have

$$
\begin{aligned}
& \psi\left(d\left(h_{1}\left(x_{2}\right), h_{2}\left(x_{3}\right)\right)\right) \\
& \quad \leq \sqrt{\gamma\left(\psi\left(d\left(h_{1}\left(x_{1}\right), h_{2}\left(x_{2}\right)\right)\right)\right)} \psi\left(d\left(h_{1}\left(x_{1}\right), h_{2}\left(x_{2}\right)\right)\right) \\
& \quad \leq \sqrt{\gamma\left(\psi\left(d\left(h_{1}\left(x_{1}\right), h_{2}\left(x_{2}\right)\right)\right)\right)} \sqrt{\gamma\left(\psi\left(d\left(h_{1}\left(x_{0}\right), h_{2}\left(x_{1}\right)\right)\right)\right)} \psi\left(d\left(h_{1}\left(x_{0}\right), h_{2}\left(x_{1}\right)\right)\right) .
\end{aligned}
$$

By $(8)$ and $\sqrt{\gamma\left(\psi\left(d\left(h_{1}\left(x_{0}\right), h_{2}\left(x_{1}\right)\right)\right)\right)}<1$, we have

$$
\psi\left(d\left(h_{1}\left(x_{1}\right), h_{2}\left(x_{2}\right)\right)\right) \leq \psi\left(d\left(h_{1}\left(x_{0}\right), h_{2}\left(x_{1}\right)\right)\right) .
$$

The function $\gamma$ is increasing, by (10), we obtain

$$
\psi\left(d\left(h_{1}\left(x_{2}\right), h_{2}\left(x_{3}\right)\right)\right) \leq\left(\sqrt{\gamma\left(\psi\left(d\left(h_{1}\left(x_{0}\right), h_{2}\left(x_{1}\right)\right)\right)\right)}\right)^{2} \psi\left(d\left(h_{1}\left(x_{0}\right), h_{2}\left(x_{1}\right)\right)\right) .
$$

Again, by (9),

$$
d\left(h_{1}\left(x_{2}\right), h_{2}\left(x_{3}\right)\right) \leq\left(\sqrt{\gamma\left(\psi\left(d\left(h_{1}\left(x_{0}\right), h_{2}\left(x_{1}\right)\right)\right)\right)}\right)^{2} d\left(h_{1}\left(x_{0}\right), h_{2}\left(x_{1}\right)\right)
$$

manifestly, $h_{1}\left(x_{2}\right) \neq h_{2}\left(x_{3}\right)$. Take

$$
q_{2}=\frac{\left(\sqrt{\gamma\left(\psi\left(d\left(h_{1}\left(x_{0}\right), h_{2}\left(x_{1}\right)\right)\right)\right)}\right)^{2} \psi\left(d\left(h_{1}\left(x_{0}\right), h_{2}\left(x_{1}\right)\right)\right)}{\psi\left(d\left(h_{1}\left(x_{2}\right), h_{2}\left(x_{3}\right)\right)\right)} .
$$

Then $q_{2}>1$. If $h_{2}\left(x_{3}\right) \in S x_{3}$, then $x_{3}$ is a coincidence point of $h_{2}$ and $S$. Assume that $h_{2}\left(x_{3}\right) \notin$ $S x_{3}$. Then

$$
0<\psi\left(d\left(h_{2}\left(x_{3}\right), S x_{3}\right)\right) \leq \psi\left(H\left(T x_{2}, S x_{3}\right)\right)<q_{2} \psi\left(H\left(T x_{2}, S x_{3}\right)\right) .
$$


Thus there exists $h_{1}\left(x_{4}\right) \in S x_{3}$ such that $\left(h_{2}\left(x_{3}\right), h_{1}\left(x_{4}\right)\right) \in E(\Gamma)$ and

$$
\begin{aligned}
\psi\left(d\left(h_{2}\left(x_{3}\right), h_{1}\left(x_{4}\right)\right)\right)< & q_{2} \psi\left(H\left(T x_{2}, S x_{3}\right)\right) \\
\leq & q_{2} \gamma\left(\psi\left(P\left(h_{1}\left(x_{2}\right), h_{2}\left(x_{3}\right)\right)\right)\right) \psi\left(P\left(h_{1}\left(x_{2}\right), h_{2}\left(x_{3}\right)\right)\right) \\
& +q_{2} L \phi\left(Q\left(h_{1}\left(x_{2}\right), h_{2}\left(x_{3}\right)\right)\right) .
\end{aligned}
$$

Similarly, $P\left(h_{1}\left(x_{2}\right), h_{2}\left(x_{3}\right)\right) \leq d\left(h_{1}\left(x_{2}\right), h_{2}\left(x_{3}\right)\right)$ and $Q\left(h_{1}\left(x_{2}\right), h_{2}\left(x_{3}\right)\right)=0$.

So, by (12),

$$
\begin{aligned}
& \psi(\left.d\left(h_{2}\left(x_{3}\right), h_{1}\left(x_{4}\right)\right)\right) \\
& \leq \sqrt{\gamma\left(\psi\left(d\left(h_{1}\left(x_{2}\right), h_{2}\left(x_{3}\right)\right)\right)\right)} \psi\left(d\left(h_{1}\left(x_{2}\right), h_{2}\left(x_{3}\right)\right)\right) \\
& \leq \sqrt{\gamma\left(\psi\left(d\left(h_{1}\left(x_{2}\right), h_{2}\left(x_{3}\right)\right)\right)\right)}\left(\sqrt{\gamma\left(\psi\left(d\left(h_{1}\left(x_{0}\right), h_{2}\left(x_{1}\right)\right)\right)\right)}\right)^{2} \\
& \quad \times \psi\left(d\left(h_{1}\left(x_{0}\right), h_{2}\left(x_{1}\right)\right)\right) .
\end{aligned}
$$

By $(11)$ and ${\sqrt{\gamma\left(\psi\left(d\left(h_{1}\left(x_{0}\right), h_{2}\left(x_{1}\right)\right)\right)\right.}}^{2}<1$, we have

$$
\psi\left(d\left(h_{1}\left(x_{2}\right), h_{2}\left(x_{3}\right)\right)\right) \leq \psi\left(d\left(h_{1}\left(x_{0}\right), h_{2}\left(x_{1}\right)\right)\right)
$$

Again, $\gamma$ is increasing, so using (13),

$$
d\left(h_{2}\left(x_{3}\right), h_{1}\left(x_{4}\right)\right) \leq\left(\sqrt{\gamma\left(\psi\left(d\left(h_{1}\left(x_{0}\right), h_{2}\left(x_{1}\right)\right)\right)\right)}\right)^{3} d\left(h_{1}\left(x_{0}\right), h_{2}\left(x_{1}\right)\right) .
$$

It is clear that $h_{2}\left(x_{3}\right) \neq h_{1}\left(x_{2}\right)$. Put

$$
q_{3}=\frac{\left(\sqrt{\gamma\left(\psi\left(d\left(h_{1}\left(x_{0}\right), h_{2}\left(x_{1}\right)\right)\right)\right)}\right)^{3} \psi\left(d\left(h_{1}\left(x_{0}\right), h_{2}\left(x_{1}\right)\right)\right)}{\psi\left(d\left(h_{1}\left(x_{2}\right), h_{2}\left(x_{3}\right)\right)\right)} .
$$

Then $q_{3}>1$. Continuing this process, we construct a sequence $\left\{h\left(x_{n}\right)\right\}$ in $M$ such that

$$
\begin{aligned}
& h_{1}\left(x_{2 n}\right) \in S x_{2 n-1}, \quad h_{2}\left(x_{2 n-1}\right) \in T\left(x_{2 n-2}\right) \\
& \left(h_{1}\left(x_{2 n-2}\right), h_{1}\left(x_{2 n-1}\right)\right),\left(h_{1}\left(x_{2 n-1}\right), h_{1}\left(x_{2 n}\right)\right) \in E(\Gamma) .
\end{aligned}
$$

Define the sequence $\left\{h\left(x_{n}\right)\right\}$ as follows:

$$
h\left(x_{n}\right)= \begin{cases}h_{1}\left(x_{n}\right), & n \text { is even } \\ h_{2}\left(x_{n}\right), & n \text { is odd }\end{cases}
$$

Let $t=\sqrt{\gamma\left(\psi\left(d\left(h_{1}\left(x_{0}\right), h_{2}\left(x_{1}\right)\right)\right)\right)}$, then $0<t<1$. We have

$$
d\left(h\left(x_{n}\right), h\left(x_{n+1}\right)\right) \leq t^{n} d\left(h_{1}\left(x_{0}\right), h_{2}\left(x_{1}\right)\right)
$$

Since $0<t<1$, we have

$$
\sum_{n=0}^{\infty}\left(d\left(h\left(x_{n}\right), h\left(x_{n+1}\right)\right)\right) \leq d\left(h_{1}\left(x_{0}\right), h_{2}\left(x_{1}\right)\right) \sum_{n=0}^{\infty} t^{n}<\infty .
$$


Thus, the sequence $\left\{h\left(x_{n}\right)\right\}$ is Cauchy in $(M, d)$. Since $M$ is complete, the sequence $h\left(x_{n}\right)$ converge to point $w$ for some $w \in M$. Let $u, v \in M$ with $h_{1}(u)=w=h_{2}(v)$. By (iii), there exists $\left\{h\left(x_{n_{k}}\right)\right\}$ such that $\left(h\left(x_{n_{k}}\right), h_{1}(u)\right) \in E(\Gamma)$ for any $n \in \mathbb{N}$. We assert that $h_{1}(u) \in T u$ or $h_{2}(v) \in S v$.

Let $\mathcal{A}=\left\{n_{k} \mid n_{k}\right.$ is even $\}$ and $\mathcal{B}=\left\{n_{k} \mid n_{k}\right.$ is odd $\}$. Then obviously $\mathcal{A} \cup \mathcal{B}$ is infinite and so at least $\mathcal{A}$ or $\mathcal{B}$ must be infinite. In the case that $\mathcal{A}$ is infinite, for each $h_{2}\left(x_{n_{k}+1}\right), n_{k} \in \mathcal{A}$, we have

$$
\begin{aligned}
D\left(h_{2}(v), S(v)\right) \leq & d\left(h_{2}(v), h_{2}\left(x_{n_{k}+1}\right)\right)+D\left(h_{2}\left(x_{n_{k}+1}\right), S(v)\right) \\
\leq & d\left(h_{2}(v), h_{2}\left(x_{n_{k}+1}\right)\right)+H\left(T\left(x_{n_{k}}\right), S(v)\right) \\
\leq & d\left(h_{2}(v), h_{2}\left(x_{n_{k}+1}\right)\right)+\gamma\left(\psi\left(P\left(h_{1}\left(x_{n_{k}}\right), h_{2}(v)\right)\right)\right) \psi\left(P\left(h_{1}\left(x_{n_{k}}\right), h_{2}(v)\right)\right) \\
& +L \phi\left(Q\left(h_{1}\left(x_{n_{k}}\right), h_{2}(v)\right)\right) \\
= & d\left(h_{2}(v), h_{2}\left(x_{n_{k}+1}\right)\right)+\gamma\left(\psi\left(d\left(h_{1}\left(x_{n_{k}}\right), h_{1}(v)\right)\right)\right) \psi\left(d\left(h_{1}\left(x_{n_{k}}\right), h_{2}(v)\right)\right),
\end{aligned}
$$

where

$$
\begin{aligned}
P\left(h_{1}\left(x_{n_{k}}\right), h_{2}(v)\right)= & \max \left\{d\left(h_{1}\left(x_{n_{k}}\right), h_{2}(v)\right), D\left(h_{1}\left(x_{n_{k}}\right), T x_{n_{k}}\right), D\left(h_{2}(v), S v\right),\right. \\
& \left.\frac{D\left(h_{1}\left(x_{n_{k}}\right), T x_{n_{k}}\right)+D\left(h_{2}(v), S v\right)}{2}\right\} \text { and } \\
Q\left(h_{1}\left(x_{n_{k}}\right), h_{2}(v)\right)= & \min \left\{D\left(h_{1}\left(x_{n_{k}}\right), S v\right), D\left(h_{2}(v), T x_{n_{k}}\right)\right\} .
\end{aligned}
$$

With $n \rightarrow \infty$, we get $Q\left(h_{1}\left(x_{n_{k}}\right), h_{2}(v)\right)=0$ and with regard to (14) we obtain

$$
\psi\left(D\left(h_{2}(v), S(v)\right)\right) \leq \gamma\left(\psi\left(h_{2}(v), S(v)\right)\right) \psi\left(D\left(h_{2}(v), S(v)\right)\right)<\psi\left(D\left(h_{2}(v), S(v)\right)\right),
$$

which is a contradiction, unless $D\left(h_{2}(v), S(v)\right)=0$. Since $T v$ is closed, thus $h_{2}(v) \in S(v)$. Similarly, we can prove that $h_{1}(v) \in T(v)$ when $\mathcal{B}$ is infinite. This completes the proof. We notice also that if both $\mathcal{A}, \mathcal{B}$ are infinite, then $h_{1}(v) \in T(v)$ and $h_{2}(v) \in S(v)$.

Example 2.3 Let $M=[0,1]$ and $d$ be the standard metric on $M$. Let $\Gamma=(V(\Gamma), E(\Gamma))$ be a directed graph with $V(\Gamma)=M$ and

$$
E(\Gamma)=\left\{(x, x),\left(0, \frac{1}{4}\right),\left(\frac{1}{4}, 0\right),\left(0, \frac{1}{16}\right),\left(\frac{1}{16}, 0\right),\left(\frac{1}{4}, \frac{1}{16}\right),\left(\frac{1}{16}, \frac{1}{4}\right): x \in M\right\} .
$$

Let $T: M \rightarrow P_{b, c l}(M)$ be defined by

$$
\begin{aligned}
& T x= \begin{cases}\left\{\frac{1}{4}\right\} & \text { if } x=1, \\
\left\{0, \frac{1}{4}\right\} & \text { if } x \in(0,1)-\left\{\frac{1}{4}, \frac{1}{2}\right\}, \\
\left\{\frac{1}{16}\right\} & \text { if } x \in\left\{0, \frac{1}{4}, \frac{1}{2}\right\},\end{cases} \\
& S x= \begin{cases}\left\{\frac{1}{16}\right\} & \text { if } x=0, \frac{1}{16}, \frac{1}{4}, 1, \\
\left\{0, \frac{1}{16}\right\} & \text { if } x \in(0,1)-\left\{\frac{1}{16}, \frac{1}{4}\right\} .\end{cases}
\end{aligned}
$$


Let $h_{1}, h_{2}: M \rightarrow M$ be defined by $h_{1}(x)=x^{2}, h_{2}(x)=x$. Consider $\psi(t)=t$ and $\gamma(t)=\frac{t+1}{t+2}$. Then it is evident that $S, T$ are mixed Geraghty-type $G$-multi-valued respect to $h_{1}$, $h_{2}$. Note that (i), (ii) and (iii) of Theorem 2.2 hold. Besides, if $(h(x), h(y)) \in E(\Gamma)$, then $H(T h(x), T h(y))=0$. Hence, for all $x, y \in M$ we have $(h(x), h(y)) \in E(\Gamma)$, ergo

$$
\psi(H(S x, T y)) \leq \gamma\left(\psi\left(d\left(h_{1}(x), h_{2}(y)\right)\right)\right) \psi\left(d\left(h_{1}(x), h_{2}(y)\right)\right),
$$

for $x, y \in M$ with $\left(h_{1}(x), h_{2}(y)\right) \in E(\Gamma)$.

If $x, y \in M$ with $\left(h_{2}(x), h_{1}(y)\right) \in E(\Gamma)$, then

$$
\psi(H(S x, T y)) \leq \gamma\left(\psi\left(d\left(h_{2}(x), h_{1}(y)\right)\right)\right) \psi\left(d\left(h_{2}(x), h_{1}(y)\right)\right) .
$$

By Theorem 2.2, there exist $u, v \in M$ such that $h_{1}(u) \in T u$ or $h_{2}(v) \in S v$. In this example, $u=\frac{1}{4}$ or $u=\frac{1}{16}$.

If in Theorem 2.2, we set $h_{1}=h_{2}=h$, then we get the following corollary.

Corollary 2.4 Let $(M, d)$ be a complete metric space with the directed graph $\Gamma, h: M \rightarrow M$ is surjective map and $S, T: M \rightarrow P_{b, c l}(M)$ be h-graph preserving with

$$
\psi(H(S x, T y)) \leq \gamma(\psi(d(h(x), h(y)))) \psi(d(h(x), h(y))),
$$

for all $x, y \in M$ with $(h(x), h(y)) \in E(\Gamma)$. Suppose

(i) there exists $x_{0} \in M$ such that $\left(h\left(x_{0}\right), u\right) \in E(\Gamma)$ for some $u \in T x_{0}$;

(ii) for any sequence $\left\{x_{n}\right\}_{n \in \mathbb{N}}$ in $M$, if $x_{n} \rightarrow x$ and $\left(x_{n}, x_{n+1}\right) \in E(\Gamma)$ for $n \in \mathbb{N}$, then there is a subsequence $\left\{x_{n_{k}}\right\}_{n_{k} \in \mathbb{N}}$ such that $\left(x_{n_{k}}, x\right) \in E(\Gamma)$ for $n_{k} \in \mathbb{N}$.

Then there exist $u, v \in M$ such that $h(u) \in$ Tu or $h(v) \in S v$.

Definition 2.5 Let $(M, d)$ be a metric space endowed with a partial order $\leq$. For each $M_{1}, M_{2} \in M, M_{1} \preceq M_{2}$ if $\omega_{1} \leq \omega_{2}$ for any $\omega_{1} \in M_{1}, \omega_{2} \in M_{2}, h: M \rightarrow M$ a surjective map, and $T: M \rightarrow P_{b, c l}(M)$. $T$ is said to be $h$-increasing if for any $x, y \in M, h(x) \leq h(y)$ implies $T x \preceq T y$.

Theorem 2.6 Let $(M, d)$ be a complete metric space with partially order $\leq, h: M \rightarrow M$ be a surjective map and $T: M \rightarrow P_{b, c l}(M)$ be a multi-valued mapping. Suppose that

(i) $T$ is h-increasing;

(ii) there exist $x_{0} \in M$ and $u \in T x_{0}$ such that $h\left(x_{0}\right) \leq u$;

(iii) for each sequence $x_{k}$ such that $h\left(x_{k}\right) \leq h\left(x_{k+1}\right), k \in \mathbb{N}$ and $h\left(x_{k}\right)$ converges to $h(x)$ for some $x \in M$, then $h\left(x_{k}\right) \leq h(x)$;

(iv) for $x, y \in M$ with $h(x) \leq h(y)$, we have

$$
\psi(H(T x, T y)) \leq \gamma(\psi(d(h(x), h(y)))) \psi(d(h(x), h(y))),
$$

where $\gamma \in \mathcal{F}$ and $\psi, \phi \in \psi$.

Then there is $u \in M$ so that $h(u) \in T u$. In addition, if $h$ is injective, then there is a unique $u \in M$ such that $h(u) \in T u$. 
Proof Let $\Gamma=(V(\Gamma), E(\Gamma))$, be a graph with $V(\Gamma)=M$ and

$$
E(\Gamma)=\{(x, y) \mid x \leq y\}
$$

let $(h(x), h(y)) \in E(\Gamma)$, then $h(x) \leq h(y)$ and by (i), $T x \preceq T y$. For each $u \in T x, v \in T y$, we have $u \leq v$, thus $(u, v) \in E(\Gamma)$. That is, $T$ is $h$-graph preserving. By (ii), there exist $x_{0} \in M$ and $u \in T x_{0}$ such that $h\left(x_{0}\right) \leq u$. So $(h(x), u) \in E(\Gamma)$ and hence the property (i) in Corollary 2.4 is satisfied. Moreover, we obtain the property (ii) of Corollary 2.4 from the assumption (iii). Set $S=T$, then the $S, T$ are $h$-graph preserving mappings and fulfill

$$
\psi(H(T x, T y)) \leq \gamma(\psi(d(h(x), h(y)))) \psi(P(h(x), h(y))),
$$

for all $x, y \in M$ with $(h(x), h(y)) \in E(\Gamma)$. By Corollary 2.4 we get $h(u) \in T u$ for some $u \in M$.

Now, in addition, we suppose that $h$ is injective. Let $u, v \in M$ be such that $h(u) \in T u$ and $h(v) \in T v$. Suppose, on the contrary, that $h(u) \neq h(v)$. We assume, without loss of generality, that $h(u)<h(v)$. Since $h(u) \in T u$ and $h(v) \in T v$, it yields $D(h(u), T u)=D(h(v), T v)$ and hence

$$
\begin{aligned}
\psi(d(h(u), h(v))) & \leq \psi(H(T u, T v)) \leq \gamma(\psi(d(h(u), h(v)))) \psi(d(h(u), h(v))), \\
& <\psi(d(h(u), h(v))) .
\end{aligned}
$$

This leads to a contradiction. Thus $h(u)=h(v)$. Since $h$ is injective, we have $u=v$.

Corollary 2.7 Let $(M, d)$ be a complete metric space endowed with a graph $\Gamma=(V(\Gamma)$, $E(\Gamma)), S, T: M \rightarrow M$ be generalized mappings such that

$$
\psi(d(S x, T y)) \leq \gamma(\psi(d(x, y))) \psi(d(x, y))
$$

\section{mapping in $(M, d)$. Suppose that}

(i) $\exists x_{0} \in M$ such that $\left(x_{0}, u\right) \in E(\Gamma)$ for some $u \in T x_{0}$,

(ii) if $(x, y) \in E(\Gamma)$, then $(e, f) \in E(\Gamma)$ for all $e \in T x$ and $f \in$ Sy and if $(x, y) \in E(\Gamma)$, then $(w, r) \in E(\Gamma)$ for all $w \in S x$ and $r \in T y$,

(iii) for $\left\{x_{n}\right\}_{n \in \mathbb{N}}$ in $M$, if $x_{n} \rightarrow x$ and $\left(x_{n}, x_{n+1}\right) \in E(\Gamma)$ for $n \in \mathbb{N}$, then there is a subsequence $\left\{x_{n_{k}}\right\}_{n_{k} \in \mathbb{N}}$ such that $\left(x_{n_{k}}, x\right) \in E(\Gamma)$ for $n_{k} \in \mathbb{N}$.

Then there exist $u, v \in M$ such that $u \in T u$ or $v \in S v$.

\section{Application}

In this section, we apply our theorem for a solution of the following integral system:

$$
\left\{\begin{array}{l}
x(t)=\int_{a}^{b} K(t, s) f(t, s, x(s), y(s)) d s+h(t), \\
y(t)=\int_{a}^{b} K(t, s) f(t, s, y(s), x(s)) d s+h(t), \quad t \in[a, b],
\end{array}\right.
$$

where $X:=C([a, b], \mathbb{R})$ with $\|x\|_{\infty}=\sup _{t \in[a, b]}|x(t)|$, for $x \in C([a, b], \mathbb{R})$.

Let $G$ be a graph, defined by $V(\Gamma)=X$, and

$$
E(\Gamma)=\{(x, y) \in X \times X: x(t) \leq y(t) \text { or } y(t) \leq x(t) \text { for } t \in[a, b]\} .
$$


Let $d$ be the metric induced by the norm. It follows that $(X, d)$ is a complete metric space endowed with a directed graph $\Gamma$. Define mappings $S, T: X \rightarrow X$ by

$$
\left\{\begin{array}{l}
S x(t)=\int_{a}^{b} K(t, s) f(t, s, x(s), y(s)) d s+h(t), \\
T y(t)=\int_{a}^{b} K(t, s) f(t, s, y(s), x(s)) d s+h(t),
\end{array}\right.
$$

where $x, y \in C([a, b], \mathbb{R})$.

\section{Theorem 3.1 Consider Eq. (16) and suppose that}

1. $f:[a, b] \times[a, b] \times \mathbb{R} \times \mathbb{R} \rightarrow \mathbb{R}$ is continuous function and there exists $M>0$ such that $\max _{t \in[a, b]} \int_{a}^{b} K(t, s) d s<M$.

2 .

$$
|f(t, s, y(s), x(s))-f(t, s, x(s), y(s))| \leq \frac{1}{\alpha} \frac{|y(s)-x(s)|^{2}}{1+|y(s)-x(s)|}
$$

3. There exists a function $x_{0} \in X$ such that

$$
x_{0}(t) \leq \int_{a}^{b} K(t, s) f\left(t, s, x_{0}(s), T x_{0}(s)\right) d s+h(t), \quad t \in[a, b] .
$$

4. The inequality $x(s) \leq y(s)$ implies $T x(s) \leq S y(s)$ and $S x(s) \leq T y(s)$.

5. For $\left\{x_{n}\right\}_{n \in \mathbb{N}}$ in $M$, if $x_{n} \rightarrow x$ and $x_{n}(t) \leq x_{n+1}(t)$ for $n \in \mathbb{N}$, then there is a subsequence $\left\{x_{n_{k}}\right\}_{n_{k} \in \mathbb{N}}$ such that $x_{n_{k}}(t) \leq x(t)$ for $n_{k} \in \mathbb{N}, t \in[a, b]$.

Then there exist $u, v \in X$ such that $u(t)=\int_{a}^{b} K(t, s) f(t, s, u(s), v(s)) d s+h(t)$ or $v(t)=$ $\int_{a}^{b} K(t, s) f(t, s, v(s), u(s)) d s+h(t)$.

Proof Let $x, y \in X$, using (2), we get

$$
\begin{aligned}
|S x(t)-T y(t)| & =\left|\int_{a}^{b} K(t, s) f(t, s, x(s), y(s)) d s-\int_{a}^{b} K(t, s) f(t, s, y(s), x(s)) d s\right| \\
& \leq \sup _{t \in[a, b]}|f(t, s, x(s), y(s))-f(t, s, y(s), x(s))| M \\
& \leq \frac{1}{M} \frac{|x(s)-y(s)|^{2}}{1+|x(s)-y(s)|} M=\frac{|x(s)-y(s)|^{2}}{1+|x(s)-y(s)|} \\
& =\gamma(|x(s)-y(s)|)|x(s)-y(s)|,
\end{aligned}
$$

where $\gamma(t)=\frac{t}{t+1}$. With setting $\psi(t)=t$, the condition 15 in Corollary 2.7 holds. With considering (3) and (4) from assumption of theorem and by definition of the graph $\Gamma$ we deduce the conditions (i) and (ii) of in Corollary 2.7 hold. Also by the condition (5) from assumption of theorem, the condition (iii) of Corollary 2.7 holds. As a result, we have

$$
\begin{aligned}
& u(t)=\int_{a}^{b} K(t, s) f(t, s, u(s), v(s)) d s+h(t), \quad \text { or } \\
& v(t)=\int_{a}^{b} K(t, s) f(t, s, v(s), u(s)) d s+h(t) .
\end{aligned}
$$

Now we use the results of our findings to solve fractional differential equations. 
Definition $3.2([22,26])$ The Riemann-Liouville fractional integral of order $\alpha>0$ of a continuous function $f:(0,+\infty) \rightarrow(-\infty,+\infty)$ is given by

$$
I_{0^{+}}^{\alpha} f(\varsigma)=\frac{1}{\Gamma(\alpha)} \int_{0}^{\varsigma}(\varsigma-\eta)^{\alpha-1} f(\eta) d \eta
$$

provided the right-hand side is pointwise defined on $(0,+\infty)$.

Definition $3.3([22,26])$ The Riemann-Liouville fractional derivative of order $\alpha>0$ of a continuous function $f:(0,+\infty) \rightarrow(-\infty,+\infty)$ is given by

$$
D_{0^{+}}^{\alpha} f(\varsigma)=\frac{1}{\Gamma(n-\alpha)}\left(\frac{d}{d t}\right)^{n} \int_{0}^{\varsigma}(\varsigma-\eta)^{n-\alpha-1} f(\eta) d \eta
$$

where $n=[\alpha]+1,[\alpha]$ denotes the integer part of the number $\alpha$, provided that the righthand side is pointwise defined on $(0,+\infty)$.

In this paper we discuss the local existence and uniqueness of positive solutions for the following coupled system of fractional boundary value problem subject to integral boundary conditions:

$$
\left\{\begin{array}{l}
D_{0^{+}}^{\alpha} u(\varsigma)+f(\varsigma, u(\varsigma), v(\varsigma))=0, \\
D_{0^{+}}^{\alpha} v(\varsigma)+f(\varsigma, v(\varsigma), u(\varsigma))=0, \quad 0<\varsigma<1, \\
u(0)=0, \quad u(1)=\int_{0}^{1} \phi(\varsigma) u(\varsigma) d \varsigma, \\
v(0)=0, \quad v(1)=\int_{0}^{1} \psi(\varsigma) v(\varsigma) d \varsigma,
\end{array}\right.
$$

where $1<\alpha \leq 2, \phi, \psi \in L^{1}[0,1]$ are nonnegative and $f \in C([0,1] \times[0, \infty),[0, \infty))$ and $D$ is the standard Riemann-Liouville fractional derivative. The functions $\phi(\varsigma), \psi(\varsigma)$ satisfy the following conditions:

(Q) $\phi, \psi:[0,1] \rightarrow[0,+\infty) \quad$ with $\phi, \psi \in L^{1}[0,1]$

and

$$
\begin{aligned}
& \sigma_{1}:=\int_{0}^{1} \phi(\varsigma) \varsigma^{\alpha-1} d \varsigma \\
& \sigma_{2}:=\int_{0}^{1} \varsigma^{\alpha-1}(1-\varsigma) \phi(\varsigma) d \varsigma, \quad \varsigma \in(0,1) .
\end{aligned}
$$

Lemma 3.4 ([29]) If $\int_{0}^{1} \phi(\varsigma) \varsigma^{\alpha-1} d \varsigma \neq 1$, then for any $\sigma \in C[0,1]$, the unique solution of the following boundary value problem:

$$
\left\{\begin{array}{l}
D_{0^{+}}^{\alpha} u(\varsigma)+\sigma(\varsigma)=0, \quad 0<\varsigma<1, \\
u(0)=0, \quad u(1)=\int_{0}^{1} \phi(\varsigma) u(\varsigma) d \varsigma,
\end{array}\right.
$$

is given by

$$
u(\varsigma)=\int_{0}^{1} G_{1 \alpha}(\varsigma, \eta) \sigma(\eta) d \eta
$$


where

$$
\begin{aligned}
& G_{\alpha}(\varsigma, \eta)=G_{1 \alpha}(\varsigma, \eta)+G_{2 \alpha}(\varsigma, \eta), \\
& G_{1 \alpha}(\varsigma, \eta)=\frac{1}{\Gamma(\alpha)}\left\{\begin{array}{l}
\varsigma^{\alpha-1}(1-\eta)^{\alpha-1}-(\eta-\varsigma)^{\alpha-1}, \\
\varsigma^{\alpha-1}(1-\eta)^{\alpha-1},
\end{array}\right. \\
& G_{2 \alpha}(\varsigma, \eta)=\frac{\varsigma^{\alpha-1}}{1-\int_{0}^{1} \phi(\varsigma) \varsigma^{\alpha-1} d \varsigma} \int_{0}^{1} \phi(\varsigma) G_{1 \alpha}(\varsigma, \eta) d \varsigma .
\end{aligned}
$$

Then $G_{\alpha}(\varsigma, \eta)$ is a Green's function.

Lemma 3.5 ([29]) Let $\alpha \in(1,2]$. Assume that $(Q)$ holds. Then the functions $G_{1 \alpha}(\varsigma, \eta)$ have the following property:

$$
\frac{(\alpha-1) \sigma_{2} \eta(1-\eta)^{\alpha-1} \varsigma^{\alpha-1}}{\left(1-\sigma_{1}\right) \Gamma(\alpha)} \leq G_{\alpha} \leq \frac{(1-\eta)^{\alpha-1} \varsigma^{\alpha-1}}{\Gamma(\alpha)\left(1-\sigma_{1}\right)} .
$$

Lemma 3.6 ([29]) Assume that (Q) holds and $f(y, x, y)$ continuous, then $(u, v) \in X \times X$ is a solution of the system (17) if and only if it is a solution of the integral equations

$$
\left\{\begin{array}{l}
u(y)=\int_{0}^{1} G_{\alpha}(y, x) f(x, u(x), v(x)) d x, \\
v(y)=\int_{0}^{1} G_{\alpha}(y, x) f(x, v(x), u(x)) d x .
\end{array}\right.
$$

Define mappings $S, T: X \rightarrow X$ by

$$
\left\{\begin{array}{l}
S u(y)=\int_{0}^{1} G_{\alpha}(y, x) f(x, u(x), v(x)) d x, \\
T v(y)=\int_{0}^{1} G_{\alpha}(y, x) f(x, v(x), u(x)) d x .
\end{array}\right.
$$

Theorem 3.7 Consider Eq. (17) and suppose that

1. $f:[0,1] \times[0, \infty) \times[0, \infty) \rightarrow \mathbb{R}$ is continuous function such that

$$
|f(t, s, y(s), x(s))-f(t, s, x(s), y(s))| \leq \frac{(1-s)^{\alpha-1} \zeta^{\alpha-1}}{\Gamma(\alpha)} \frac{|y(s)-x(s)|^{2}}{1+\mid y(s)-x(s)} .
$$

2. There exists a function $x_{0} \in X$ such that

$$
x_{0}(t) \leq \int_{a}^{b} K(t, s) f\left(t, s, x_{0}(s), T x_{0}(s)\right) d s, \quad t \in[a, b] .
$$

3. The inequality $x(s) \leq y(s)$ implies $T x(s) \leq S y(s)$ and $S x(s) \leq T y(s)$.

4. For $\left\{x_{n}\right\}_{n \in \mathbb{N}}$ in $M$, if $x_{n} \rightarrow x$ and $x_{n}(t) \leq x_{n+1}(t)$ for $n \in \mathbb{N}$, then there is a subsequence $\left\{x_{n_{k}}\right\}_{n_{k} \in \mathbb{N}}$ such that $x_{n_{k}}(t) \leq x(t)$ for $n_{k} \in \mathbb{N}, t \in[a, b]$.

Then there exist $u, v \in X$ such that

$$
\begin{aligned}
& u(t)=\int_{a}^{b} G_{\alpha}(t, s) f(t, s, u(s), v(s)) d s \quad \text { or } \\
& v(t)=\int_{a}^{b} G_{\alpha}(t, s) f(t, s, v(s), u(s)) d s .
\end{aligned}
$$


Proof Let $x, y \in X$, using (2), we get

$$
\begin{aligned}
|S x(t)-T y(t)| & =\left|\int_{a}^{b} G_{\alpha}(t, s) f(t, s, x(s), y(s)) d s-\int_{a}^{b} G_{\alpha}(t, s) f(t, s, y(s), x(s)) d s\right| \\
& \leq \sup _{t \in[a, b]}|f(t, s, x(s), y(s))-f(t, s, y(s), x(s))| \frac{\Gamma(\alpha)}{(1-s)^{\alpha-1} \varsigma^{\alpha-1}} \\
& \leq \frac{(1-s)^{\alpha-1} \varsigma^{\alpha-1}}{\Gamma(\alpha)} \frac{|x(s)-y(s)|^{2}}{1+|x(s)-y(s)|} \frac{\Gamma(\alpha)}{(1-s)^{\alpha-1} \varsigma^{\alpha-1}}=\frac{|x(s)-y(s)|^{2}}{1+|x(s)-y(s)|} \\
& =\gamma(|x(s)-y(s)|)|x(s)-y(s)|,
\end{aligned}
$$

where $\gamma(t)=\frac{t}{t+1}$. With setting $\psi(t)=t$, the condition (15) in Corollary 2.7 holds. With considering (3) and (4) from the assumption of the theorem and by definition of the graph $\Gamma$ we deduce the conditions (i) and (ii) in Corollary (2.7) hold. Also by the condition (5) from assumption of theorem, the condition (iii) of Corollary 2.7 holds. As a result, we have

$$
\begin{aligned}
& u(t)=\int_{a}^{b} G_{\alpha}(t, s) f(t, s, u(s), v(s)) d s \text { or } \\
& v(t)=\int_{a}^{b} G_{\alpha}(t, s) f(t, s, v(s), u(s)) d s .
\end{aligned}
$$

\section{Acknowledgements}

The authors thank their universities.

\section{Funding}

We declare that funding is not applicable for our paper.

\section{Research data}

No data were used to support this study.

\section{Availability of data and materials}

It is not applicable for our paper.

\section{Competing interests}

The authors declare that they have no competing interests.

\section{Authors' contributions}

Writing-review and editing were done by HA, MA and EK. All authors contributed equally and significantly in writing this article. All authors have read and agreed to the published version of the manuscript.

\section{Author details}

${ }^{1}$ Department of Mathematics, Faculty of Basic Science, University of Bonab, Bonab, Iran. ${ }^{2}$ Institute of Research and Development, Duy Tan University, 550000 Da Nang, Vietnam. ${ }^{3}$ Faculty of Natural Sciences, Duy Tan University, 550000 Da Nang, Vietnam. ${ }^{4}$ Department of Mathematics, Cankaya University, Mimar Sinan Caddesi, 06790 Ankara, Turkey.

${ }^{5}$ Department of Medical Research, China Medical University, Hsueh-Shih Road, Taichung, 40402, Taiwan.

\section{Publisher's Note}

Springer Nature remains neutral with regard to jurisdictional claims in published maps and institutional affiliations.

Received: 23 May 2020 Accepted: 3 July 2020 Published online: 14 July 2020

\section{References}

1. Abdeljawad, T., Agarwal, R.P., Karapinar, E., Kumari, P.S.: Solutions of he nonlinear integral equation and fractional differential equation using the technique of a fixed point with a numerical experiment in extended b-metric space. Symmetry 11, 686 (2019)

2. Adiguzel, R.S., Aksoy, U., Karapinar, E., Erhan, I.M.: On the solution of a boundary value problem associated with a fractional differential equation. Math. Methods Appl. Sci. (2020). https://doi.org/10.1002/mma.6652

3. Afshari, A., Atapour, M., Aydi, H.: Generalized $(\alpha-\psi)$ Geraghty multivalued mappings on b-metric spaces endowed with a graph. TWMS J. Appl. Eng. Math. 77(2), 248-260 (2017) 
4. Afshari, H., Aydi, H., Karapinar, E.: Existence of fixed points of set-valued mappings in b-metric spaces. East Asian Math. J. 32(3), 319-332 (2016)

5. Afshari, H., Aydi, H., Karapinar, E.: On generalized $\alpha-\psi$-Geraghty contractions on b-metric spaces. Georgian Math. J. 27(1), 9-21 (2020)

6. Afshari, H., Kalantari, S., Karapinar, E.: Solution of fractional differential equations via coupled fixed point. Electron. J. Differ. Equ. 2015, 286 (2015)

7. Afshari, H., Rezapour, S., Shahzad, N.: Absolute retract of of the common fixed points set of two multi-functions. Topol. Methods Nonlinear Anal. 40, 429-436 (2012)

8. Agarwal, R.P., Karapınar, E., O'Regan, D., Roldán-López-de-Hierro, A.F.: Fixed Point Theory in Metric Type Spaces. Springer, Berlin (2015)

9. Atangana, A.: Blind in a commutative world: simple illustrations with functions and chaotic attractors. Chaos Solitons Fractals 114, 347-363 (2018)

10. Atangana, A., Qureshi, S.: Modeling attractors of chaotic dynamical systems with fractal fractional operators. Chaos Solitons Fractals 123, 320-337 (2019)

11. Czerwik, S.: Nonlinear set-valued contraction mappings in b-metric spaces. Atti Semin. Mat. Fis. Univ. Modena 46(2), 263-276 (1998)

12. Echenique, F:: A short and constructive proof of Tarski's fixed point theorem. Int. J. Game Theory 33, 215-218 (2005)

13. Espinola, R., Kirk, W.A.: Fixed point theorems in R-trees with applications to graph theory. Topol. Appl. 153, 1046-1055 (2006)

14. Gulyaz-Ozyurt, S.: On some alpha-admissible contraction mappings on Branciari b-metric spaces. Adv. Theory Nonlinear Anal. Appl. 1(1), 1-13 (2017)

15. Gulyaz-Ozyurt, S.: A fixed point theorem for extended large contraction mappings. Results Nonlinear Anal. 1(1), 46-48 (2018)

16. Gulyaz-Ozyurt, S.: A note on Kannan type mappings with a F-contractive iterate. Results Nonlinear Anal. 2(3), 143-146 (2019)

17. Jachymsk, J: The contraction principle for mappings on a metric space with a graph. Proc. Am. Math. Soc. 136(4), 1359-1373 (2008)

18. Karapinar, E.: Fixed point theorems on $\alpha-\psi$-Geraghty contraction type mappings. Filomat 28(2), 761-766 (2014)

19. Karapinar, E.: $\alpha-\psi$-Geraghty contraction type mappings and some related fixed point results. Filomat 28(1), 37-48 (2014)

20. Karapinar, E., Abdeljawad, T., Jarad, F.: Applying new fixed point theorems on fractional and ordinary differential equations. Adv. Differ. Equ. 2019, 421 (2019)

21. Karapinar, E., Samet, B.: Generalized $\alpha$ - $\psi$-contractive type mappings and related fixed point theorems with applications. Abstr. Appl. Anal. 2012, Article ID 793486 (2012)

22. Kilbas, A.A., Srivastava, H.M., Trujillo, J.J.: Theory and Applications of Fractional Differential Equations. North-Holland Mathematics Studies, vol. 204, pp. 7-10 (2006)

23. Marasi, H.R., Afshari, H., Zhai, C.B.: Some existence and uniqueness results for nonlinear fractional partial differential equations. Rocky Mt. J. Math. 47, 571-585 (2017)

24. Mlaiki, N., Mohamed Hajji, M., Abdeljawad, T.: Fredholm type integral equation in extended Mb-metric spaces. Adv. Differ. Equ. 2020, 289 (2020). https://doi.org/10.1186/s13662-020-02752-4

25. Panda, S.K., Karapınar, E., Atangana, A.: A numerical schemes and comparisons for fixed-point results with applications to the solutions of Volterra integral equations in dislocated extended b-metric space. Alex. Eng. J. 59, 815-827 (2020)

26. Podlubny, I.: Fractional Differential Equations. Academic Press, New York (1999)

27. Popescu, O.: Some new fixed point theorems for $\alpha$-Geraghty contraction type maps in metric spaces. Fixed Point Theory Appl. 2014(1), 190 (2014)

28. Tiammee, J.S., Suantai, S.: Coincidence point theorems for graph-preserving multi-valued mappings. Fixed Point Theory Appl. 2014(1), 70 (2014)

29. Yang, C., Zhai, C., Zhang, L.: Local uniqueness of positive solutions for a coupled system of fractional differential equations with integral boundary conditions. Adv. Differ. Equ. 2017, 282 (2017)

\section{Submit your manuscript to a SpringerOpen ${ }^{\circ}$ journal and benefit from:}

- Convenient online submission

- Rigorous peer review

- Open access: articles freely available online

- High visibility within the field

- Retaining the copyright to your article

Submit your next manuscript at $\gg$ springeropen.com 